\title{
UN NUEVO ANALISIS DE LA DISTRIBUCION VERTICAL DE PARACALANUS CRASSIROSTRIS (COPEPODA CALANOIDEA) MEDIANTE EL MODELO LINEAR GENERAL (OMEGA)
}

\author{
ANA MILSTEIN
}

Museo Nacional de Historia Natural, Montevideo, Uruguay

\section{SYNOPSIS}

The material anaiysed here is part of a collection made with a 91 van Dorn bottle in a $5 \mathrm{~m}$ depth station at Ubatuba - Säo Pauto, between June 1976 and May 1977. Two sets of general linear models were constructed, one for the winter months (June, July and August) and the other for the summer ones (December, January and February). In each set. copepodid stages and adults were studied separately. in relation to hour of sampling, depth, interaction hour-depth, and environmental factors light penetration, dissolved oxygen, salinity and temperature, considered as a whole). All the stages studied were spread in the water column and show no vertical migration. The hour was not significant for any stage except for copepodid $V$ female in winter. This results agree with a previous analysis of the same material performed with the data of each two months throughout the year (Milstein, 1978).

\section{Introducción}

En un trabajo anterior (Milstein, 1978) se aplicó el modelo linear (Omega) al estudio de la distribución vertical de Paracalanus crassirostris durante un año, reuniendo los datos de cada dos colectas efectuadas con un mes de intervalo. Al reunir así los datos se disminuyeron las variaciones debidas a diferencias del padrón de distribución vertical durante un día en los distintos meses, y se evidenciaron las tendencias generales de la especie en un lapso relativamente corto (dos meses). Continuando con esa línea, en el presente trabajo se estudian tendencias más generales, a saber, la distribución de $P$. crassirostris en invierno y verano. Esta especie fue elegida por ser una de las circumtrópico-subtropicales de aguas costeras más abundantes.

\section{Material y Metodos}

El material utilizado corresponde a las muestras de junio, julio y agosto (invierno) y diciembre, enero y febrero (verano) de una serie colectada en once días distribuídos en el período junio 1976-maio 1977, con intervalos de 4 a 7 semanas. Dichas muestras fueron colectadas en la Enseada do Flamengo, Ubatuba - São Paulo, en una estación fija de $5 \mathrm{~m}$ de profundidad.

El área es una baía protegida, sin afluentes importantes de agua dulce. La marea es semidiurna y las aguas son eumixohalinas con temperaturas entre $20^{\circ}$ y $30^{\circ} \mathrm{C}$. En invierno llueve frecuentemente y las salinidades registradas fueron más bajas que en verano, presentándose bastante homogénea la columna da agua. En verano los gradientes de salinidad, temperatura y oxígeno disuelto entre superficie y fondo son mayores (Teixeira, 1973; Milstein, 1978). Las colectas fueron reahizadas cuatro veces cada día (a las $06.00,12.00,18.00$ y 24.00 horas) en tres niveles: superficie, $2 \mathrm{~m}$ y cerca del fondo. En cada estación, y cada nivel, fueron colectados 991 de agua con botella van Dorn de 91 y agua para determinación đe salinidad y oxígeno disuelto, y se midieron la temperatura y el porcentage de penetracion de luz. Las muestras colectadas con botellá van Dorn fueron luego filtradas por una red de 37 micras de abertura de malla, y fijadas en formol $4 \%$ neutralizado; fueron contados todos los individuos de $P$. crassirostris, cada fase de desarrollo por separado.

\section{Metodos de Analisis de Datos}

La aplicación del modelo linear general a la biología fue tratada por Seal (1964); en biología marina fue aplicado por Buzas $(1969,1971)$ y por Milstein $(1978)$; en este último trabajo podrán encontrarse más detalles sobre la metodología empleada.

El modelo (Omega) fue construido a priori tomando en consideración densidad de animales por muestra, variables ambientales (penetración de luz, salinidad, températura, oxígeno disuelto), diferencias debidas a la hora de colecta, diferencias debidas a la profundidad, e interacción entre diferencias debidas a hora y profundidad. En notación matricial, este modelo se escribe:

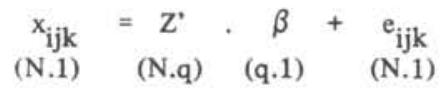

donde $x$ es un vector de $N$ observaciones; $j=1,2,3,4$ horas; $j=1,2,3$ profundidades; $\mathrm{k}=1,2,3$ réplicas (meses); $\mathrm{Z}$ es una matriz de variables instrumentalis y covariables, cuya composición se encuentra en la Tabla I; $\beta$ es un vector de q parámetros para "explicar" las $\mathrm{N}$ observaciones; $y$ e es un vector de errores (o residuos) no explicados por el modelo, que se asume sea $\mathrm{N}\left(\mathrm{O}, \hat{\sigma}^{2}\right)$. La estimativa de la variancia $\left(\hat{\sigma}^{2}\right)$ que gobierna la distribución de $\mathrm{e}_{\mathrm{ij}}$ está dada por $\mathrm{S}_{\Omega} /(\mathrm{N}-\mathrm{q})$, donde $\mathrm{S}_{\Omega}$ es la sumatoria de los cuadrados de los residuos del modelo Omega.

TABLA

Composición de la Matriz $Z$

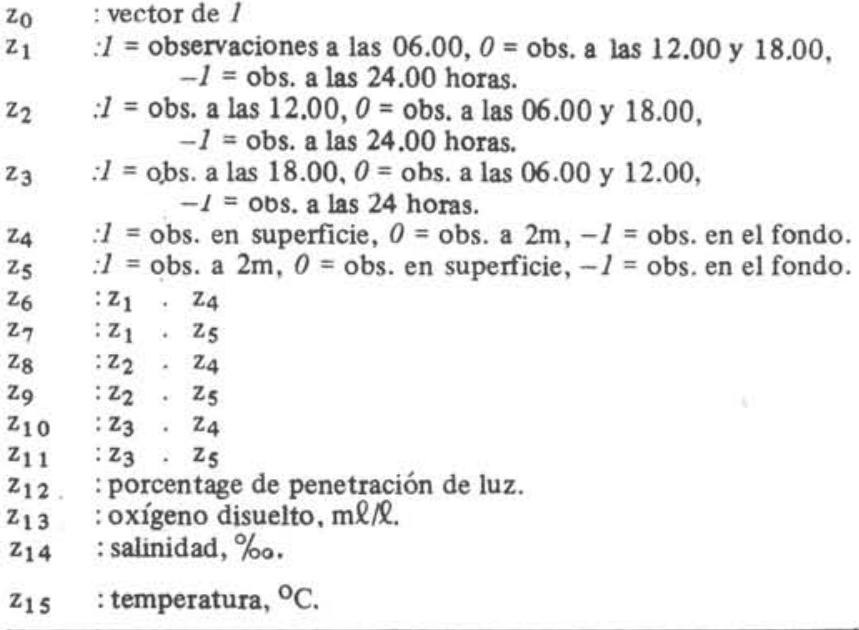

Para saber que parámetros son significativamente diferentes de cero, se comparó el modelo Omega con cuatro modelos omega restringidos (Tabla II), cuya fórmola es la misma que la de modelo Omega pero la matriz $Z^{\prime}$ consta sólo de s columnas y el vector $\beta$ de $s$ líneas. Así, cada modelo omega elimina q-s parámetros del modelo general Omega.

TABLA II

Modelos omega testados

$\begin{array}{lll}\omega & \text { Hipótesis } \beta_{1}=0 & \text { Efecto } \\ 1 & \mathrm{i}=1,2,3 . & \text { hora } \\ 2 & \mathrm{i}=4,5 . & \text { profundidad } \\ 3 & \mathrm{i}=6, \ldots, 11 . & \text { interacción hora profundidad. } \\ 4 & \mathrm{i}=12,13,14,15 . & \text { factores ambientales reunidos. }\end{array}$

La comparación entre cada modelo omega con el Omega se realiza por medio del cociente.

$$
\mathrm{F}_{\mathrm{q}-\mathrm{s}, \mathrm{N}-\mathrm{q}}=\frac{\left(\mathrm{S}_{\omega}-\mathrm{s}_{\Omega}\right) /(\mathrm{q}-\mathrm{s})}{\mathrm{S}_{\Omega} /(\mathrm{N}-\mathrm{q})}
$$




\section{Resultados}

Las pruebas de $\chi^{2}$ indicaron buena adherencia de los mo- delos Omega (Tabla III); en todos los casos, la probabilidad de obtener un valor tan grande como los hallados es mayor de $99 \%$, para 20 grados de libertad.

\section{TABLA III
$\chi^{2}$ de los modelos Omega (20 g. 1.)}

\begin{tabular}{lcccccccc} 
& henbras & machos & CVmach. & CVhemb. & CIV & CIII & CII & CI \\
inv. & 1.621 & 1.745 & 2.961 & 1.142 & 2.199 & 3.097 & 3.784 & 4.822 \\
ver. & 1.533 & 4.540 & 4.834 & 3.901 & 4.666 & 6.293 & 4.216 & 3.437 \\
\hline
\end{tabular}

Los resultados de las comparaciones de los cuatro modelos omega con el modelo Omega se encuentran en la Tabla IV. De ella se desprende que los adultos y copepoditos de P.crassirostris se distribuyen homogeneamente en toda la columna de agua y no pre- sentan migración vertical, ni en invierno ni en verano.

El factor hora fue significativo sólo para los C V hembras en invierno. En todos los demás casos, las variaciones entre un mes y otro fueron eliminadas al agrupar los datos de a tres meses, mos-

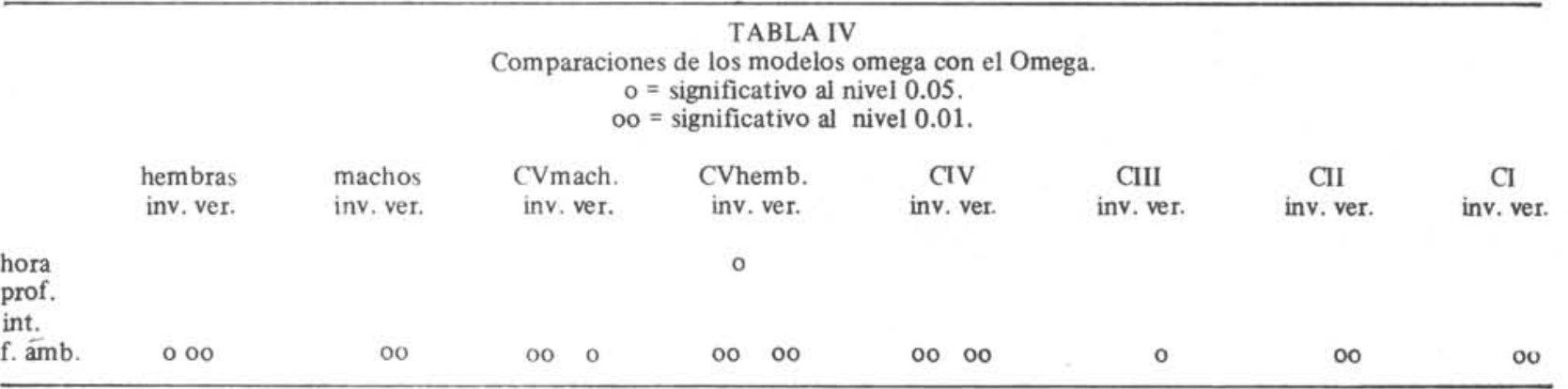

trando una tendencia mas general que indica que la cantidad de individuos capturados no fue afectada por la hora en que se realizó la colecta, en ninguna de las épocas estudiadas.

Las variables ambientales en conjunto afectaron significativamente la distribución de esta especie, en verano a todos los copepoditos y adultos y en invierno solamente a los animales mayores (copepoditos IV, V y adultos).

\section{Discusion y Conclusiones}

En el trabajo anterior (Milstein, 1978), en el que se emplearon los datos agrupados de a dos meses, los efectos de la profundidad $\mathrm{e}$ interacción hora-profundidad no fueron tan nítidos como en el presente trabajo, pero permitieron llegar a las mismas conclusiones (distribución homogénea en la columna de agua y ausencia de migración vertical).

En relación a la hora, en aquel trabajo se sugirió que en los casos en que este factor mostró significancia, se debía más a los sistemas de corrientes (mareas) que a la hora en sí. En el presente estudio no se disponen de nuevos datos para afirmar que dichas significancias se debieran realmente a la marea, pero la hora mostró no ser la responsable, salvo para los $\mathrm{C} \mathrm{V}$ hembras en invierno. Esta significancia anómala no ha podido ser explicada.

La importancia de los factores ambientales en los grupos de meses de verano para los copepoditos y adultos ya había sido señalada en el estudio anterior; ni en el presente trabajo ni en el anterior fueron encontrados valores significativos en invierno para los copepoditos menores.

Concluyendo, las tendencias observadas en el estudio de datos de dos meses a lo largo del año fueron confirmadas al analizar los datos globales de tres meses de verano y tres meses de invierno.

\section{Agradecimientos}

Agradezco a la Dra. M. Scintila de Almeida Prado por la orientación en el programa de muestreo y por el uso de equipos e instalaciones del Instituto Oceanográfico de la Universidad de San Pablo.

\section{Referencias Bibliográficas}

BUZAS, M.A. 1969. Foraminiferal species densities and environmental variables in an estuary. Limnol. Oceanogr., 14: $411-422$.

------ 1971. Analysis of species densities by the multivariate general linear model. Limnol. Oceanogr., 16: 667-670.

MILSTEIN L.. A.R. 1978. Distribuição vertical de Paracalanus crassirostris (Copepoda Calanoidea). Analise mediante um modelo linear. Dissertação de Mestrado. Universidade de São Paulo, Instituto Oceanográfico. 35p. (en inglés: Bolm Inst. oceanogr., S Paulo, 1979, 28 (2): 65-78.

SEAL, H. L. 1964. Multivariate statistical analysis for biologists. London, Mathuen, 207p.

TEIXEIRA, C. 1973. Preliminary studies of primary production in the Ubatuba region (Lat. $25^{\circ} 30^{\prime} \mathrm{S}$ - Long. $45^{\circ} 06^{\prime} \mathrm{W}$. Brazil. Bolm Inst. oceanogr., S Paulo, 22: 49-58. 